\section{Case Reports in Oncology}

\title{
Sequential Therapy with Nivolumab Followed by Ipilimumab Induces Complete Response in Metastatic Melanoma of the Lung but with Severe Hepatotoxicities
}

\author{
Sadanori Furudate Taku Fujimura Yumi Kambayashi Takanori Hidaka \\ Akira Hashimoto Setsuya Aiba \\ Department of Dermatology, Tohoku University Graduate School of Medicine, \\ Sendai, Japan
}

\section{Keywords}

Nivolumab · Ipilimumab · Metastatic melanoma · Immune-related adverse event · Immune checkpoint inhibitors

\section{Abstract}

Since nivolumab significantly prolongs survival in patients with metastatic melanoma, the number of patients administered nivolumab is increasing, but only $30-40 \%$ of patients who received nivolumab monotherapy experienced objective tumor regression. Therefore, enhancing its anti-tumor immune response is of great interest to dermato-oncologists. In this report, we present a case of multiple metastatic melanomas in the lung successfully treated with nivolumab ( $2 \mathrm{mg} / \mathrm{kg}$ every 3 weeks for 12 weeks) followed by ipilimumab ( $3 \mathrm{mg} / \mathrm{kg} \mathrm{ev}$ ery 3 weeks for 9 weeks), but with severe liver dysfunction. 
 Oncology}

\section{Introduction}

The PD-1/PD-L1 pathway plays a critical role in the tumor immune response in melanoma [1-4]. Nivolumab, an anti-PD-1 antibody, significantly prolonged survival in patients with metastatic melanoma, but only $30-40 \%$ of the patients who received nivolumab monotherapy experienced objective tumor regression $[2,5,6]$. Since nivolumab significantly prolongs survival in patients with metastatic melanoma, enhancing its anti-tumor immune response is of great interest to dermato-oncologists [5, 7, 8]. Indeed, Weber et al. [9] recently reported the efficacy of sequential administration of nivolumab followed by ipilimumab and its clinical benefits.

In this report, we present a case of multiple metastatic melanomas in the lung successfully treated with nivolumab ( $2 \mathrm{mg} / \mathrm{kg}$ every 3 weeks for 12 weeks) followed by ipilimumab ( $3 \mathrm{mg} / \mathrm{kg}$ every 3 weeks for 9 weeks), but with severe liver dysfunction. Our case suggests that the anti-tumor immune response and immune-related adverse events (irAE) might develop in a dose-independent manner by immune checkpoint inhibitors.

\section{Case Report}

An 80-year-old Japanese man visited our outpatient clinic with a red, easy-to-bleed nodule on his scalp. On his initial visit, physical examination revealed a red-brown, dome-shaped nodule on his scalp (fig. 1a). A biopsy specimen revealed dense infiltration of spindle-shaped atypical cells with pigmentation from the epidermis to the deep dermis (fig. 1b). From the above findings, we diagnosed this patient as having malignant melanoma, excised the tumor, and performed left cervical lymph node dissection. In addition, after the surgical treatment, the patient was treated with continuous administration of interferon- $\beta\left(3.0 \times 10^{6} \mathrm{U}\right)$ for 10 days, once a month for 6 times, followed by the administration of interferon- $\beta$ (3.0 $\times$ $10^{6} \mathrm{U}$ ) once a month. Two years after the surgical treatment, follow-up computed tomography (CT) revealed multiple lung metastases of melanoma (fig. 2a), which was confirmed by exfoliative cytodiagnosis using a bronchoscope. We administered nivolumab at $2 \mathrm{mg} / \mathrm{kg}$ every 3 weeks for 12 weeks. Since follow-up CT suggested progressive lung metastasis 3 weeks after the administration of nivolumab, we sequentially administered ipilimumab at $3 \mathrm{mg} / \mathrm{kg}$ every 3 weeks. Nine weeks after the administration of ipilimumab, the serum total bilirubin level had increased to 3 times the normal range. We diagnosed the patient's condition as ipilimumab-induced hepatotoxicity, based on a previous report [10]. We intravenously administered methylprednisolone sodium succinate $1 \mathrm{mg} / \mathrm{kg} /$ day with appropriate reduction, and the serum total bilirubin level decreased to within the normal range. After the administration of methylprednisolone sodium succinate, we treated the patient with oral prednisolone 40, 35, and $30 \mathrm{mg} /$ day for 1 week each. When we reduced prednisolone to $25 \mathrm{mg} /$ day, the serum AST and ALT levels suddenly increased to 3 times the normal range. We intravenously administered methylprednisolone sodium succinate $1 \mathrm{mg} / \mathrm{kg} / \mathrm{day}$ with appropriate reduction again, and the serum AST and ALT level decreased to within the normal range. After the administration of methylprednisolone sodium succinate, we treated the patient with oral prednisolone with appropriate reduction, and there was no recurrence of hepatotoxicities even when we stopped the administration of oral prednisolone. In addition, in parallel with the severe hepatotoxicities, prominent systemic vitiligo developed, suggesting the induction of anti-melanoma immune response [6] (fig. 2b). Therefore, we performed a follow-up CT scan 3 months after the administration of ipilimumab during the administra- 


\section{Case Reports in Oncology}

tion of oral prednisolone for hepatitis, which revealed a prominent regression of the lung metastasis (fig. 2a).

\section{Discussion}

We describe a case of multiple metastatic melanomas in the lung successfully treated with a complete response using sequential therapy of nivolumab $(2 \mathrm{mg} / \mathrm{kg}$ every 3 weeks for 12 weeks) followed by ipilimumab (3 mg/kg every 3 weeks for 9 weeks). In addition, in parallel with severe hepatotoxicities, prominent systemic vitiligo developed, which suggested the induction of anti-melanoma immune response [6]. Indeed, although we stopped immunotherapy for 3 months to treat the immunotherapy-induced hepatitis, the metastatic melanomas in the lung showed dramatic reduction in the tumor mass during the administration of methylprednisolone sodium succinate. Our case suggests that nivolumab followed by ipilimumab strongly induced an anti-melanoma immune response with severe irAE.

Immune checkpoint inhibitors, such as nivolumab and ipilimumab, significantly prolong the overall survival and response rate of unresectable metastatic melanoma [2, 5]. Indeed, Larkin et al. [5] reported that the response rate of nivolumab for unresectable metastatic melanoma is $33.7 \%$ and that of ipilimumab $19.0 \%$. Moreover, combined nivolumab and ipilimumab significantly improved the response rate (57.9\%), and this method is one of the most effective protocols for unresectable metastatic melanoma with or without BRAF mutation, though the ratio of irAE was also significantly increased. In addition, more recently, Weber et al. [9] reported that the anti-tumor efficacy of sequential administration of nivolumab ( $3 \mathrm{mg} / \mathrm{kg}$ every 2 weeks for 12 weeks) followed by ipilimumab (3 mg/kg every 3 weeks for 12 weeks) is superior to its reverse sequence. Notably, they also reported that there was no significant difference in the rate of treatment-related grade 3-5 adverse events in each group [10], suggesting that nivolumab followed by ipilimumab is a clinically more beneficial option compared to the reverse sequence [9].

Since nivolumab is IgG4, which has a half-life of 3 weeks in the blood, we administered nivolumab at a dose of $2 \mathrm{mg} / \mathrm{kg} 4$ times every 3 weeks, suggesting that an appropriate dose of nivolumab remained in the blood when we first administered ipilimumab at a dose of $3 \mathrm{mg} / \mathrm{kg}$. Unexpectedly, despite the fact that the blood concentration of nivolumab was lower compared with previous reports [5,9], our case developed bimodal severe hepatitis. Moreover, although we stopped the administration of nivolumab and ipilimumab during the administration of methylprednisolone sodium succinate followed by prednisolone, the melanoma in the lung decreased dramatically, suggesting that a long-term, continuous antimelanoma immune response was induced by this sequential therapy. Our case suggested that development of an anti-tumor immune response and irAE by the immune checkpoint inhibitor might develop in a dose-independent manner. Since this report presents only a single case, further cases may provide fundamental insights into the mechanisms of immune checkpoint inhibitor-induced anti-tumor immune response and irAE.

\section{Statement of Ethics}

The patient gave written informed consent. 


\section{Case Reports in Oncology}

Furudate et al: Sequential Therapy with Nivolumab Followed by Ipilimumab Induces Complete Response in Metastatic Melanoma of the Lung but with Severe Hepatotoxicities

\section{Disclosure Statement}

The authors have no conflicting financial or other interests to declare.

\section{References}

1 Hino R, Kabashima K, Kato Y, Yagi H, Nakamura M, Honjo T, Okazaki T, Tokura Y: Tumor cell expression of programmed cell death-1 ligand 1 is a prognostic factor for malignant melanoma. Cancer 2010;116: $1757-1766$.

-2 Topalian SL, Sznol M, McDermott DF, Kluger HM, Carvajal RD, Sharfman WH, Brahmer JR, Lawrence DP, Atkins MB, Powderly JD, Leming PD, Lipson EJ, Puzanov I, Smith DC, Taube JM, Wigginton JM, Kollia GD, Gupta A, Pardoll DM, Sosman JA, Hodi FS: Survival, durable tumor remission, and long-term safety in patients with advanced melanoma receiving nivolumab. J Clin Oncol 2014;32:1020-1030.

-3 Wolchok JD, Kluger H, Callahan MK, Postow MA, Rizvi NA, Lesokhin AM, Segal NH, Ariyan CE, Gordon RA, Reed K, Burke MM, Caldwell A, Kronenberg SA, Agunwamba BU, Zhang X, Lowy I, Inzunza HD, Feely W, Horak CE, Hong Q, Korman AJ, Wigginton JM, Gupta A, Sznol M: Nivolumab plus ipilimumab in advanced melanoma. N Engl J Med 2013;369:122-133.

-4 Fujimura T, Ring S, Umansky V, Mahnke K, Enk AH: Regulatory T cells stimulate B7-H1 expression in myeloid-derived suppressor cells in ret melanomas. J Invest Dermatol 2012;132:1239-1246.

5 Larkin J, Chiarion-Sileni V, Gonzalez R, Grob JJ, Cowey CL, Lao CD, Schadendorf D, Dummer R, Smylie M, Rutkowski P, Ferrucci PF, Hill A, Wagstaff J, Carlino MS, Haanen JB, Maio M, Marquez-Rodas I, McArthur GA, Ascierto PA, Long GV, Callahan MK, Postow MA, Grossmann K, Sznol M, Dreno B, Bastholt L, Yang A, Rollin LM, Horak C, Hodi FS, Wolchok JD: Combined nivolumab and ipilimumab or monotherapy in untreated melanoma. N Engl J Med 2015;373:23-34.

-6 Teulings HE, Limpens J, Jansen SN, Zwinderman AH, Reitsma JB, Spuls PI, Luiten RM: Vitiligo-like depigmentation in patients with stage III-IV melanoma receiving immunotherapy and its association with survival: a systematic review and meta-analysis. J Clin Oncol 2015;33:773-781.

-7 Kakizaki A, Fujimura T, Furudate S, Kambayashi Y, Yamauchi T, Yagita H, Aiba S: Immunomodulatory effect of peritumorally administered interferon-beta on melanoma through tumor-associated macrophages. Oncoimmunology 2015;4:e1047584.

-8 Fujimura T, Furudate S, Kakizaki A, Kambayashi Y, Haga T, Hashimoto A, Aiba S: Contact immunotherapy enhances the therapeutic effects of nivolumab in treating in-transit melanoma: two cases reports. J Dermatol 2016;43:686-689.

-9 Weber JS, Gibney G, Sullivan RJ, Sosman JA, Slingluff CL Jr, Lawrence DP, Logan TF, Schuchter LM, Nair S, Fecher L, Buchbinder EI, Berghorn E, Ruisi M, Kong G, Jiang J, Horak C, Hodi FS: Sequential administration of nivolumab and ipilimumab with a planned switch in patients with advanced melanoma (CheckMate 064): an open-label, randomised, phase 2 trial. Lancet Oncol 2016;17:943-955.

10 Weber JS, Kähler KC, Hauschild A: Management of immune-related adverse events and kinetics of response with ipilimumab. J Clin Oncol 2012;30:2691-2697. 


\section{Case Reports in Oncology}

\begin{tabular}{l|l}
\hline Case Rep Oncol 2016;9:644-649 \\
\hline DOI: 10.1159/000450974 & $\begin{array}{l}\text { @ 2016 The Author(s). Published by S. Karger AG, Basel } \\
\text { www.karger.com/cro }\end{array}$ \\
\hline
\end{tabular}

Furudate et al.: Sequential Therapy with Nivolumab Followed by Ipilimumab Induces Complete Response in Metastatic Melanoma of the Lung but with Severe Hepatotoxicities
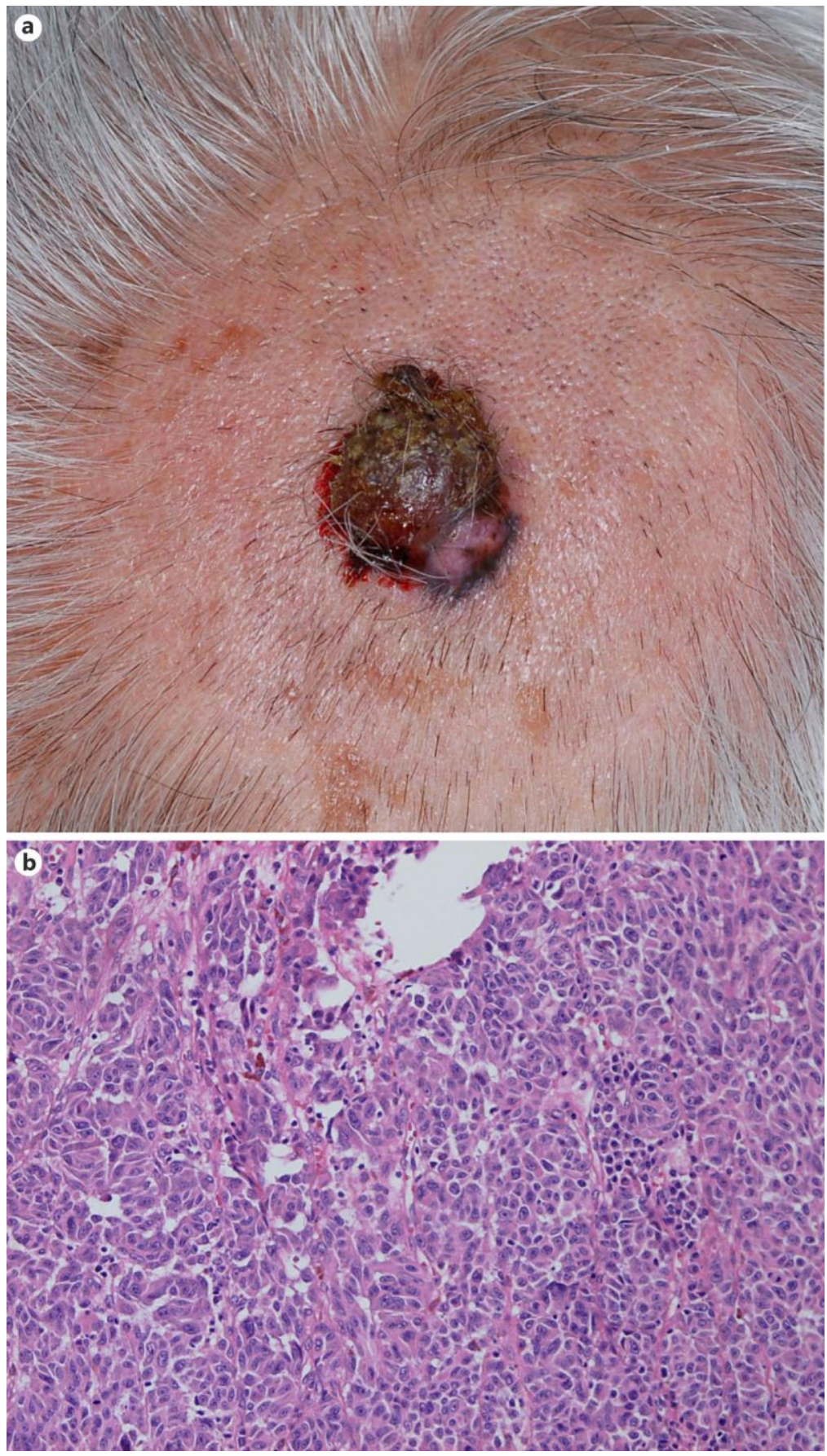

Fig. 1. a A red-brown, dome-shaped nodule on the scalp. b Dense infiltration of spindle-shaped atypical cells with pigmentation from the epidermis to the deep dermis. 


\section{Case Reports in Oncology}
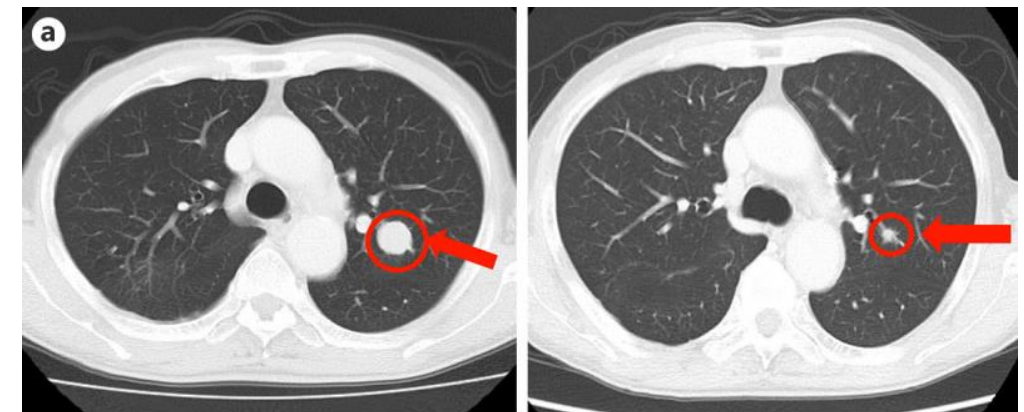

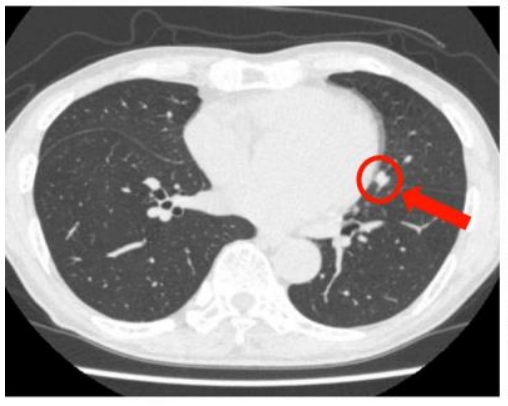

Pre-treatment

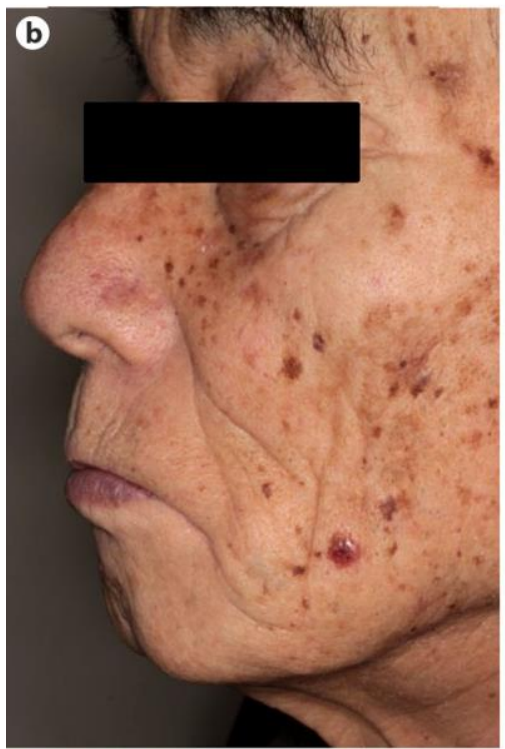

Post-treatment

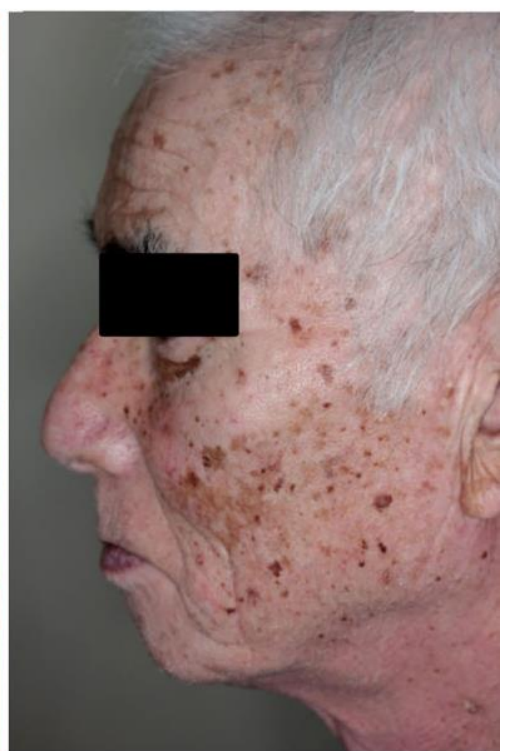

Post-treatment

Fig. 2. a Multiple lung metastases of melanoma before and after sequential therapy. b Prominent, multiple vitiligo developed on the face after sequential therapy. 